\title{
Examining nanoparticle assemblies using high spatial resolution x-ray microtomography
}

\author{
P. M. Jenneson, R. D. Luggar, and E. J. Morton \\ School of Electronics and Physical Sciences, University of Surrey, Guildford, Surrey GU2 7XH, \\ United Kingdom \\ O. Gundogdu and U. Tüzün \\ School of Engineering, University of Surrey, Guildford, Surrey GU2 7XH, United Kingdom
}

(Received 8 April 2004; accepted 8 June 2004)

\begin{abstract}
An experimental system has been designed to examine the assembly of nanoparticles in a variety of process engineering applications. These applications include the harvesting from solutions of nanoparticles into green parts, and the subsequent sintering into finished components. The system is based on an x-ray microtomography with a spatial resolution down to $5 \mu \mathrm{m}$. The theoretical limitations in x-ray imaging are considered to allow experimental optimization. A standard nondestructive evaluation type apparatus with a small focal-spot $\mathrm{x}$-ray tube, high-resolution complementary metal oxide semiconductor flat-panel pixellated detector, and a mechanical rotational stage is used to image the static systems. Dynamic sintering processes are imaged using the same x-ray source and detector but a custom rotational stage which is contained in an environmental chamber where the temperature, atmospheric pressure, and compaction force can be controlled. Three-dimensional tomographic data sets are presented here for samples from the pharmaceutical, nutraceutical, biotechnology, and nanoparticle handling industries and show the microscopic features and defects which can be resolved with the system. () 2004 American Institute of Physics. [DOI: 10.1063/1.1776635]
\end{abstract}

\section{INTRODUCTION}

The application of x-ray computed tomographic (CT) techniques to nondestructive evaluation of materials, outside of the medical field for which they were initially developed, is a rapidly expanding area of research. ${ }^{1}$ The expensive body sized medical CT machines have been replaced by relatively inexpensive bench-top sets. The typical spatial resolutions of $0.5 \mathrm{~mm}$ for medical systems have also been improved down to $5 \mu \mathrm{m}$ for bench-top sets. These improvements are mainly due to the advancement in pixellated x-ray detectors and the simultaneous development of microfocus x-ray tubes. For example, third generation (cone-beam geometry, singlesource, two-dimensional detector) medical CT systems use 12 in. x-ray image intensifiers (detector pixel pitch of 0.5 $\mathrm{mm}$ ) and $1 \mathrm{~mm}$ focal-spot size $\mathrm{x}$-ray tubes in a proximity focused geometry. Compared to a modern non-destructive evaluation (NDE) CT set which often use flat-panel pixellated caesium iodide coated complementary metal oxide semiconductor (CMOS) arrays (detector pixel pitch of $50 \mu \mathrm{m})$ and $\mathrm{x}$-ray tubes with a $5 \mu \mathrm{m}$ focal-spot size in a geometry which magnifies the sample on the detector. The use of improved detectors has the additional benefit of better contrast resolution and the signal digitization is higher (x-ray image intensifiers are digitized to 8 bits, CMOS flat-panel arrays are digitized to 12 bits). The use of NDE CT sets for use in the study of nanopowder assemblies is only just coming to fruition as the spatial and contrast resolutions are only just approaching the limits for which useful information can be obtained.

The x-ray microtomography system at the University of Surrey is currently being used to examine several powder and particulate systems. There is particular interest in the dynamic evolution of particulate systems as they are sintered and, in particular, the physical properties of the system. The bulk-density, moisture content, interparticle spacing, and particle shape are all important parameters which affect the macroscale bulk properties of the particle systems postsintering. It is this microscale particle system which is modeled in computational fluid dynamics simulations. There is currently a missing link between the microscale structure of particles as they dynamically evolve during sintering and the way in which this affects the bulk properties. It is proposed to image this microscale system using $\mathrm{x}$-ray microtomography, reduce the complexity of this raw 14-bit level data using geometrical reasoning, which can then be inputted into computational fluid dynamics simulations to ascertain the bulk properties, thus closing the information loop. The appearance of fractures and large pores in the green part (i.e., presintering) has detrimental effects on its mechanical properties, such as yield strength and hardness, as well as in some cases affecting thermal and electrical conductive properties. The current research is focused on a better understanding of the microstructural evolution of the particle assembly during a coupled uniaxial compaction/drying process of wet particle clusters.

\section{THEORETICAL CONSIDERATIONS OF X-RAY TOMOGRAPHIC IMAGING}

The minimum attainable spatial resolution of a tomographic projection acquired with an $\mathrm{x}$-ray imaging system is limited by four factors: the $\mathrm{x}$-ray source size, the geometrical magnification, the detector pixel size, and the detector element pitch. The number of angular projections taken and the 
(a)

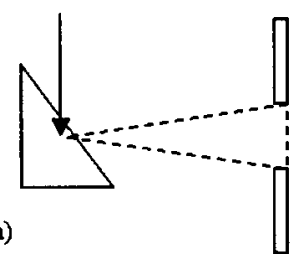

(b)

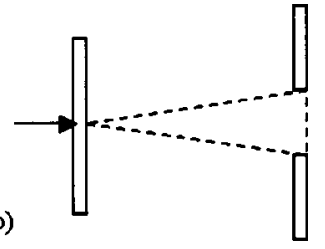

FIG. 1. Schematic of (a) reflection and (b) transmission target geometries used in $\mathrm{X}$-ray tubes.

precision in mechanically moving the sample to this position determines the spatial accuracy of the tomographic reconstruction. The accuracy of linear attenuation coefficients for each voxel calculated during the tomographic reconstruction is determined by the photon energies used during the acquisition of the projections.

\section{A. Image formation}

The imaging system used in x-ray microtomography uses exactly the same geometrical principles as shadow imaging with an optical system with an extended source. ${ }^{2}$ The spatial resolution in the formed image is determined by either the size of the extended source if high magnification is used, the detector pixel size if the sample is in close proximity to the detector, or more usually a combination of the two. It follows that at the two extremes it is either the extended photon-source size or the detector sampling which limit the spatial resolution. The smallest detector pixel sizes are obtained from a combination of charge-coupled devices with a $7 \mu \mathrm{m}$ pixel size coupled to an $\mathrm{x}$ ray to light converting screens, giving an effective pixel detector size of $1-2 \mu \mathrm{m} .{ }^{3}$ In contrast the smallest extended-source size for the formation of $\mathrm{x}$ rays is $\approx 400 \mathrm{~nm}^{4}$ It is therefore logical to use a spatially small extended-source target and a high magnification geometry, this also reduces the tolerances which need to be placed on the detector pixel size of the detector.

\section{B. X-ray source size}

Most of the current microfocus X-ray (MFX) sources are based on transmission targets. In contrast most medical and industrial $\mathrm{x}$-ray sources use a reflection target, the geometries for reflection and transmission $\mathrm{x}$-ray targets are shown in Fig. 1.

Using the continuously slowing down approximation (CSDA) range for electrons in matter, ${ }^{5}$ the maximum range for molybdenum and tungsten reflection targets can be plotted against incoming electron energy, see Fig. 2.

The focal-spot size is highly dependent on energy in the reflection geometry. The transmission geometry reduces this range at higher energies as mean free path of the electron is comparable with the target thickness and multiple scatters are less likely to occur.

Monte Carlo simulations were conducted using the GEANT v4.6.0 radiation transportation code. ${ }^{6}$ The typical thickness of a transmission target in current commercial systems is $\approx 4 \mu \mathrm{m}$. Thus, the geometry was defined as a $4 \mu \mathrm{m}$ thickness of target material with the electron source normal to surface boundary. A range of electron energies from

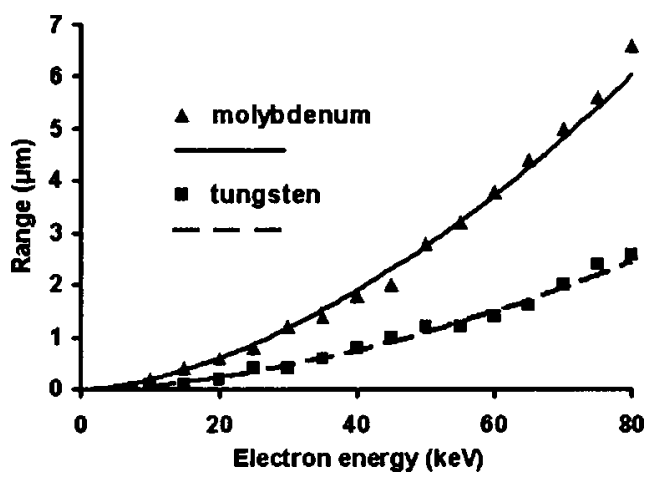

FIG. 2. Theoretical limit of focal-spot size for a reflection target based on double the CSDA range, for molybdenum and tungsten targets, for a range of electron energies from 10 to $80 \mathrm{keV}$.

$20 \mathrm{keV}$ to $80 \mathrm{keV}$ were projected into the target in a pencil beam of $\delta$ width. The distance from the input axis were recorded in intensity versus radial distance from the electron input point on the exit face of the target. The full width at half maximum of the scattering ranges for electrons in a $4 \mu \mathrm{m}$ molybdenum target for different energies are shown in Fig. 3.

The electron spread from the Monte Carlo data shows the full width at half maximum scattering range for electrons in a $4 \mu \mathrm{m}$ thick transmission molybdenum target is about $122 \mathrm{~nm}$ and is energy independent. This shows it is not electron scattering within the target which is limiting focal-spot size (cf. reflection targets) but it is more likely to be spacecharge repulsion effects in the incoming electron beam used to generate a sufficient flux of $\mathrm{x}$ rays, which limits the minimum focal-spot size.

\section{Geometrical magnification}

The finite size of the $\mathrm{x}$-ray source size limits the ultimate spatial resolution of an imaging system. ${ }^{2}$ The unsharpness $U_{g}$ associated with the focal spot of size $a$ is dependent on the magnification $m$ :

$$
U_{g}=a(1-1 / m) .
$$

The detector pixel size $F$ also introduces receptor unsharpness $U_{r}$ which when combined in quadrature with $U_{g}$ determines the overall unsharpness $U$. The optimum magnifica-

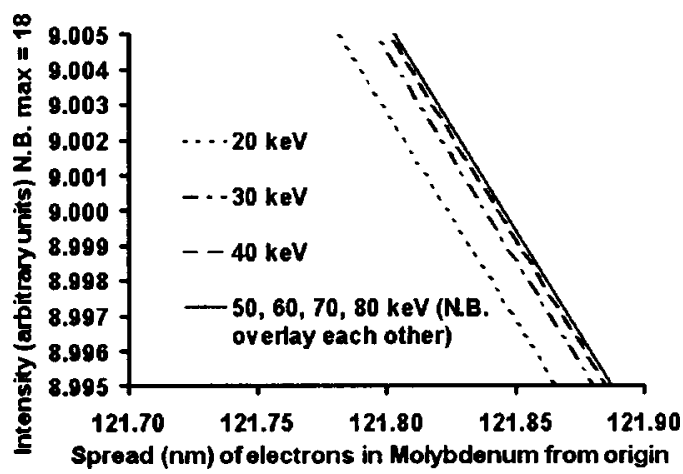

FIG. 3. Monte Carlo simulations of the electron spread in a $4 \mu \mathrm{m}$ thick transmission molybdenum target for electron energies from $20 \mathrm{keV}$ to $80 \mathrm{keV}$. 


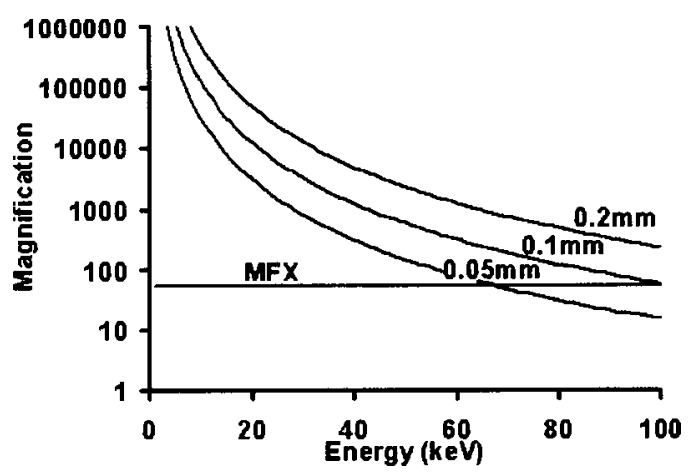

FIG. 4. The optimum magnification for imaging with a reflection target system and the optimum magnification for a $5 \mu \mathrm{m}$ focal-spot MFX with a detector aperture of $50 \mu \mathrm{m}$.

tion $m_{o}$ for a system occurs when both types of unsharpness contribute significantly to the overall unsharpness, i.e., when $(d U / d m)=0$. It follows that

$$
m_{o}=1+(c F / a)^{2},
$$

where $c$ is a constant introduced to correct for the shape of the x-ray source, for a Gaussian source $c=0.72$. The optimum magnification is plotted against energy determined from the focal-spot size for a reflection molybdenum target for detector pixel sizes of $50 \mu \mathrm{m}, 100 \mu \mathrm{m}$, and $200 \mu \mathrm{m}$, see Fig. 4.

For transmission microfocal x-ray tubes, as the focalspot size is not so dependent on the incoming electron energy, any energy will produce optimum images and it is the source to sample and sample to detector distances that need to be considered.

\section{Modulation transfer function}

The modulation transfer function (MTF) can be determined by taking the modulus of the square of the imaging system transfer function (ISTF). The ISTF as a function of spatial frequency is dependent on the $\mathrm{x}$-ray source size, the detector pixel size, and the image magnification. For a source with a Gaussian distribution and a detector consisting of many square elements, the MTF of the system is a product of a Gaussian (from the source) and a sinc function (from the detector elements):

$$
\begin{aligned}
\operatorname{MTF}_{x}(\xi)= & \mid\left(\left\{\sin \left(\pi a \xi t_{x}\right) /\left(\pi a \xi t_{x}\right)\right\}\right. \\
& \left.\times \exp \left[-\pi\left\{(1-a)^{2} \xi^{2} r_{f}^{2}\right\}\right]\right)^{2} \mid,
\end{aligned}
$$

where $\xi$ is the spatial frequency, $r_{f}$ is the source radius, $t_{x}$ is the detector pixel size, and $a$ is the reciprocal geometric magnification. The ISTF for a detector with a pixel size of $50 \mu \mathrm{m}$ is plotted for magnifications of 5,10 , and 20 and a source radius of $5 \mu \mathrm{m}$ is shown in Fig. 5. For magnifications greater than 10 there is still power in the image signal at a resolution of 200 line-pairs/mm $(\sim 5 \mu \mathrm{m})$, indicated by the positive magnitude of the ISTF. Changing the focal-spot size of the source will have virtually no effect on the MFT as it is the detector pixel size which band limits the measured image.

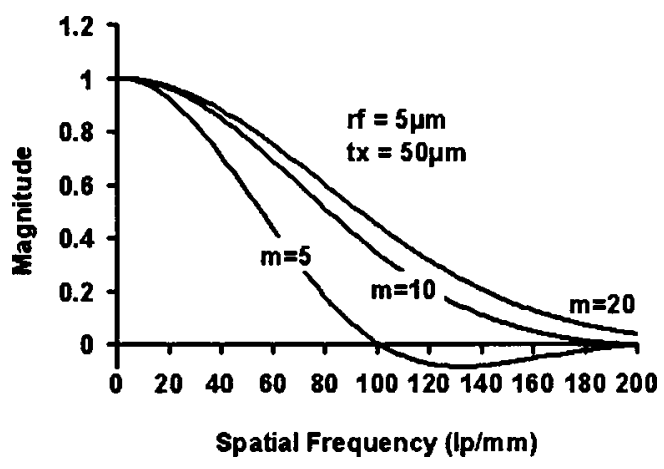

FIG. 5. The image system transfer function for magnifications of 5, 10, and 20 for an imaging system with a $5 \mu \mathrm{m}$ source size and a detector aperture size of $50 \mu \mathrm{m}$.

\section{E. Angular sampling requirements}

The number of projections needed to adequately sample Fourier space is given by the Nyquist sampling theorem ${ }^{7}$ for an object diameter $D$ with detector pixel size $t$. The number of projections needed to satisfy this is given by Eq. (4):

$$
N_{\phi}=\pi D / 2 t \text {. }
$$

The mechanical movement of the sample must be accurate to the precision required by the Nyquist sampling theorem.

\section{F. Photon energies}

The photon energies used to acquire projections have an effect on the accuracy of the calculation of linear attenuation coefficients in the reconstructed voxels. ${ }^{8}$ By combining the relationship for the noise propagated in an image [Eq. (5)] with an expression for the total run time [Eq. (6)], the accuracy of the measured attenuation coefficients can be calculated [Eq. (7)]. $\Delta \mu$ is the noise propagated in an image due to the finite photon count $n$ per ray sum for filtered back projection. $T$ is the total run time for a complete tomographic acquisition:

$$
\begin{aligned}
& \Delta \mu=k(2 \pi / n D t)^{1 / 2}, \\
& T=8 \pi n N^{4} \sinh (\mu D) / \mu D A^{\prime}, \\
& \Delta \mu / \mu=4 \pi k N^{2}\left\{[D \sinh (\mu D)] /\left[t T A^{\prime}(\mu D)^{3}\right]\right\}^{1 / 2},
\end{aligned}
$$

where $k$ is a factor dependent on the digital filter used for back projection (e.g., $k=0.289$ for a RamachandranLakshminarayanan filter), $D$ is the object diameter, $t$ the detector pixel size, $N$ is the number of detector elements, $\mu$ the linear attenuation coefficient, and $A^{\prime}$ is the photon-source intensity. If all the geometrical factors, the total run time, and the source intensity are fixed, the accuracy depends on the "sensitivity factor" $\left[(\sinh (\mu D) /(\mu D))^{3}\right]^{1 / 2}$ as shown in Fig. 6.

The optimum energy of the x-ray source can be determined from the sensitivity factor given by Eq. (7). The primary component of the image for our example is spherical particles of aluminum oxide particles close packed $\left(\mathrm{Al}_{2} \mathrm{O}_{3}\right.$, with an equivalent density of $2.05 \mathrm{~g} \mathrm{~cm}^{-3}$ ). The linear attenuation coefficients for the aluminum oxide particles for 


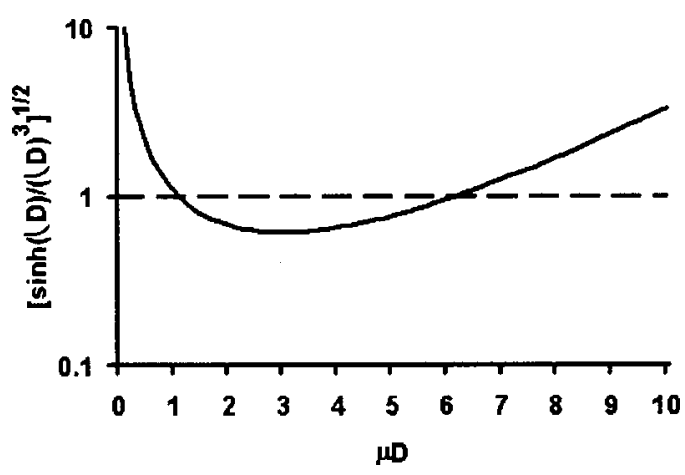

FIG. 6. Sensitivity factor for tomographic imaging with energetic photons such as $\mathrm{x}$ rays.

the energy range from 0 to $80 \mathrm{keV}$ can be determined by XCOM. ${ }^{9}$ This is then related to the MFP length in the particle assembly and plotted in Fig. 7.

The optimum energy for an assembly of close packed spherical particles of aluminum oxide with a diameter of $20 \mathrm{~mm}$ is therefore between $16 \mathrm{keV}$ and $27 \mathrm{keV}$ but optimum at $19 \mathrm{keV}$. Molybdenum has a $K_{\alpha}$ characteristic peak at $17.5 \mathrm{keV}$ which is close to the optimum energy compared to tungsten at $59.3 \mathrm{keV}$ (the usual choice of target material for a microfocus $\mathrm{x}$ ray). Hence a molybdenum target $\mathrm{x}$-ray tube was filtered with $100 \mu \mathrm{m}$ of zirconium to give the quasimonoenergetic spectrum shown in Fig. 8.

Monochromating the source has two useful effects. The dose is minimized as only radiation with an energy optimized for imaging passes through the sample. Beam hardening artefact associated with reconstructions from polychromatically illuminated projections is also eliminated.

\section{EXPERIMENTAL APPARATUS}

Figure 9 shows the experimental apparatus used for high-resolution x-ray CT. The detector is a Hamamatsu C7942 flat-panel CMOS array $(2400 \times 2400$ pixels at a $50 \mu \mathrm{m}$ pixel pitch with a CsI coating) and a Hamamatsu L6731-01 microfocus x-ray tube (Mo target $80 \mathrm{kVp}$, $100 \mathrm{~mA}, 5 \mu \mathrm{m}$ focal-spot size). The sample stage is a computer controlled rotational stepper motor with a resolution of $0.01^{\circ}$. The ring artefact commonly associated with third generation scanners (caused by the repeated use of the same detector elements in each projection) is minimized by me-

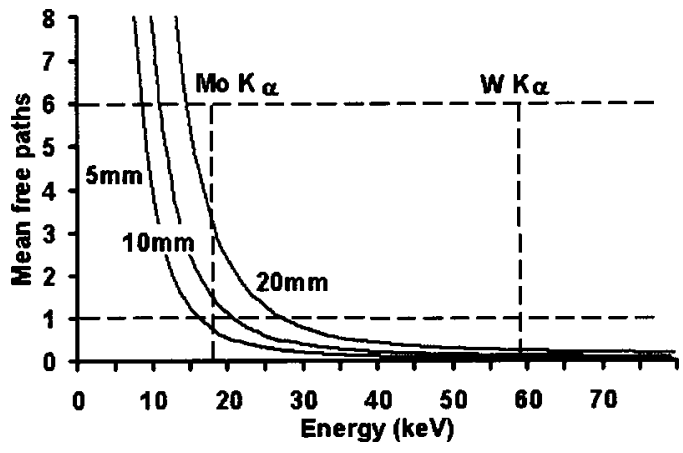

FIG. 7. MFP of $x$ rays for $5 \mathrm{~mm}, 10 \mathrm{~mm}$, and $20 \mathrm{~mm}$ diameters of close packed spherical particles of aluminum oxide. The Mo $K_{\alpha}$ and W $K_{\alpha}$ energies are also shown.

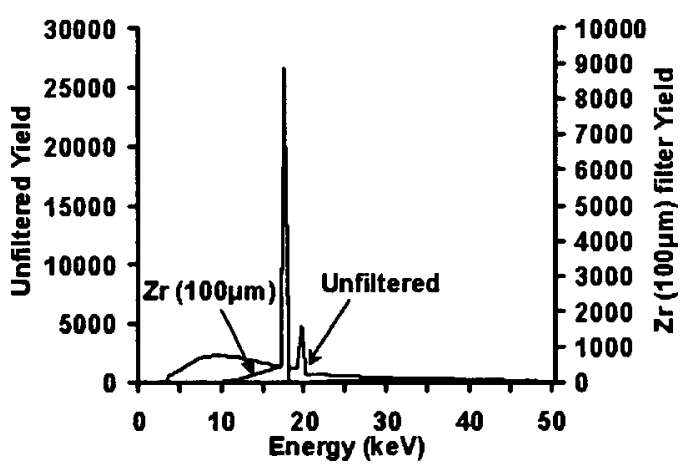

FIG. 8. Spectra from an unfiltered and a $100 \mu \mathrm{m}$ zirconium filtered molybdenum target X-ray tube operated at $50 \mathrm{kVp}$. The molybdenum $K_{\alpha}$ peaks are normalized with respect to their relative intensity scales (left unfiltered, right filtered) to overlay on the plot.

chanically translating the detector sidewards by a random integer number of pixels for each projection. The sample is rotated through $360^{\circ}$ in a number of steps determined by the Nyquist sampling theorem (typically, number of steps $=\pi / 2 \times$ number of detector elements). The projection data are then stored and at a later stage reconstructed using a Feldkamp based three-dimensional back-projection routine ${ }^{10}$ on the Beowulf parallel-processing cluster into a threedimensional data set of the linear attenuation coefficients. This can then be processed using a variety of signalprocessing methods to extract useful information from the data.

The two-dimensional projection 14-bit level data are collected on a $2048 \times 2048 \times 2048$ pixellated detector therefore producing a three-dimensional data set of $2048 \times 2048$ $\times 2048$ voxels (thus a $10 \times 10 \times 10 \mathrm{~mm}^{3}$ sample can be imaged at $5 \mu \mathrm{m}$ ). If the reconstruction data are stored as double-precision data to preserve the accuracy of the data, the data storage size is 68 gigabytes. It is therefore essential to reduce this raw data in a geometrical reasonable way in order to view and perform numerical calculations pertaining to its properties. This is even more important if the particles are to be dynamically tracked as the particle system evolves during sintering. Multiple 68 gigabytes data sets of raw data would be virtually impossible to resolve dynamically, but a simple description of individual particles and their properties would reduce the problem to a manageable task.

The normal rotational stage can be replaced by a beryllium or carbon fibre windowed environmental chamber,

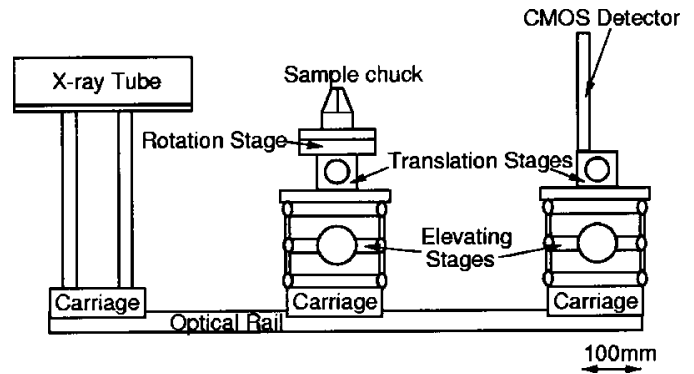

FIG. 9. Schematic diagram of the high-resolution x-ray microtomography apparatus. 


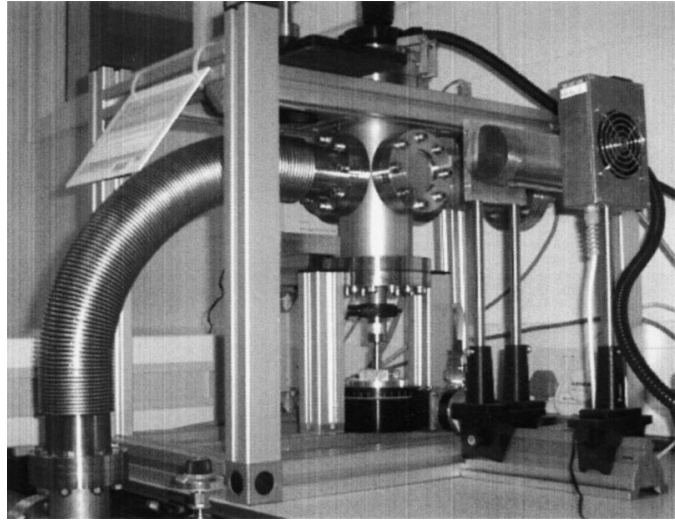

FIG. 10. Photograph of the environmental chamber rotation stage.

shown in Fig. 10. The samples can then be heated (up to $1000 \mathrm{~K}$ ) and compressed (up to $6 \mathrm{kPa}$ ) under partial pressure (1 atm to below $10^{-6}$ mbar).

\section{Applications}

$\mathrm{X}$-ray microtomography is an ideal tool for imaging nanopowder assemblies but does not have the resolution to image individual nanoparticles. Nevertheless the harvesting of large nanoparticles $(\sim 500 \mathrm{~nm})$ from colloidal suspensions is possible. ${ }^{11}$ The formation of the assembly can be studied as the suspension medium is evaporated and information regarding the packing density and large area defects can be obtained. The samples can then be mechanically tested and desirable properties can be linked to the formation and structure of the final assembly. The drying process can then be adapted allowing the evolution of better harvesting techniques.

The formation of vacuum sintered nanoparticle assemblies can also be studied and refined using the vacuumfurnace rotation stage. The sintered regime can be carefully controlled and the effects of heating cycles, axial loading impulses, and vacuum conditions can all be assessed against desirable mechanical properties.

The formation of nanoparticles assemblies has a particular application in the pharmaceutical industry. The formation of cold-pressed pills from powder assemblies can result in undesirable mechanical properties. If the compaction of the powder is too low the pill may not be stable during transport. If the compaction is too high the pill may not release the drug in the body in the most effective way. Irregularities in the internal structure of the pill therefore need to be linked to manufacturing conditions. Figure 11 shows X-ray CT data taken of a Sterwin Medicines Codydramol pill.
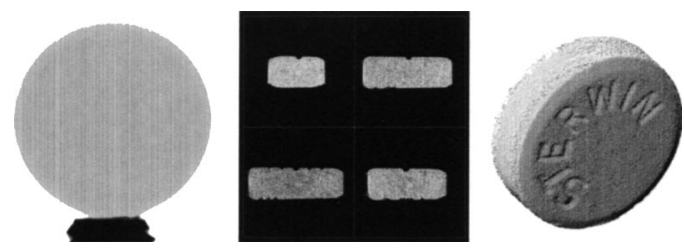

FIG. 11. An x-ray projection, reconstructed tomographic slices, and threedimensional (3D) rendered isosurface of a cold-pressed pill (N.B. pill diameter is $10 \mathrm{~mm})$

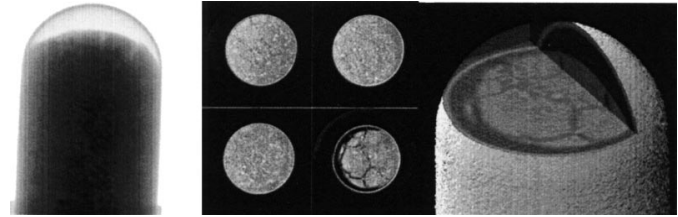

FIG. 12. An x-ray projection, reconstructed tomographic slices, and 3D rendered isosurface with a cutaway of an encapsulated powder pill (N.B. pill diameter is $6 \mathrm{~mm}$ )

If a pharmaceutical nanoparticle assembly is encapsulated and the powder is not cold pressed, then the way in which it settles during transport can affect the drug delivery system. Figure 12 shows $\mathrm{x}$-ray CT data taken of a generic loperamide hydrochloride capsule. The fissures and packing density of the powder can be seen.

Figure 13 shows x-ray CT data from a breakfast cereal. The figure shows a three-dimensional isosurface render of the outside of the cereal and the inner air voids. The number of voids and their size was determined with a threedimensional void labeling algorithm written using IDL v6.0 (Research Systems Inc., Boulder, CO). ${ }^{12}$ The ability to extract information with $\mathrm{x}$-ray microtomography, which is pertinent to engineering process control, is an important feedback mechanism for the development of more efficient and economic manufacturing processes.

\section{CONCLUSIONS}

The use of x-ray microtomography has an important role to play in the analysis of nanoparticle assemblies as it provides three-dimensional data necessary to understand how such assemblies evolve during processing. It has been shown that an x-ray microtomography system optimized from both spatial and contrast resolution produces high-quality infor-
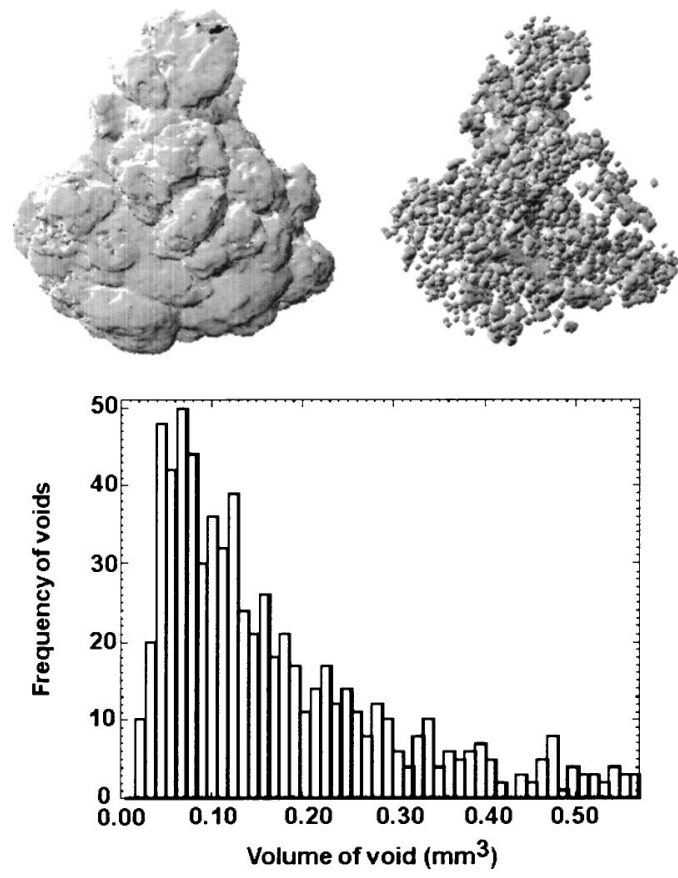

FIG. 13. Air voidation in a breakfast cereal and the frequency of the volume of these voids (N.B. approximate height of cereal is $15 \mathrm{~mm}$ ). 
mation about the internal structure of a variety of important industrial samples such as pharmaceutical and nutraceutical nanopowder assemblies. The system at the University of Surrey has been designed, based on theoretical x-ray tomographic imaging criteria, to analyze such samples in the area of nanoparticle process engineering. The additional environmental chamber holds the samples and allows the evolution of nanoparticle assemblies to be dynamically imaged using bench-top x-ray microtomography.

\section{ACKNOWLEDGMENT}

We are grateful to the Engineering and Physical Sciences Research Council in the UK for funding this work (Grant No. GR/R34370).
${ }^{1}$ J. H. Dunsmuir, S. R. Ferguson, K. L. D'Amico, B. P. Flannery, and H. W. Deckman, NDT \& E Int. 27-2, 110 (1994).

${ }^{2} \mathrm{H}$. H. Barrett and W. Swindell, Radiological Imaging, 2nd ed. (Academic, New York, 1981).

${ }^{3}$ U. Bonse, R. Nusshardt, F. Busch, R. Pahl, J. H. Kinney, Q. C. Johnson, and R. A. Saroyan, J. Mater. Sci. 26, 4076 (1991).

${ }^{4}$ See http://www.hpk.co.jp/Eng/products/ETD/mfxe/mfxe.htm

${ }^{5}$ L. Landau, J. Phys. (Moscow) 8, 201 (1944).

${ }^{6}$ S. Agostinelli et al., Nucl. Instrum. Methods Phys. Res. A 506, 250 (2003).

${ }^{7}$ A. G. Lindgren and P. A. Rattey, Digit. Signal Process. 7-4, 371 (1994).

${ }^{8}$ W. B. Gilboy, Nucl. Instrum. Methods Phys. Res. A 221, 193 (1984).

${ }^{9}$ J. H. Hubbell, Int. J. Appl. Radiat. Isot. 33, 1269 (1982).

${ }^{10}$ L. A. Feldkamp, L. C. Davis, and J. W. Kress, J. Opt. Soc. Am. A 1, 612 (1984).

${ }^{11}$ H. J. Krause, A. Schwartz, and P. Rohdewald, Drug Dev. Ind. Pharm. 12, 527 (1986).

${ }^{12}$ L. Gumley, Practical IDL Programming (Morgan Kaufmann, Los Altos, CA, 2001). 